Adaptation in virtual environments: conceptual framework and user models Peer-reviewed author version

OCTAVIA, Johanna; RAYMAEKERS, Chris \& CONINX, Karin (2011) Adaptation in virtual environments: conceptual framework and user models. In: MULTIMEDIA TOOLS AND APPLICATIONS, 54(1). p. 121-142.

DOI: $10.1007 / \mathrm{s} 11042-010-0525-\mathrm{z}$

Handle: http://hdl.handle.net/1942/12014 
1

Multimedia Tools and Applications manuscript No.

(will be inserted by the editor)

\title{
Adaptation in Virtual Environments \\ Conceptual Framework and User Models
}

\author{
Johanna Renny Octavia • Chris Raymaekers · \\ Karin Coninx
}

the date of receipt and acceptance should be inserted later

\begin{abstract}
When interacting in a virtual environment, users are confronted with a number of interaction techniques. These interaction techniques may complement each other, but in some circumstances can be used interchangeably. Because of this situation, it is difficult for the user to determine which interaction technique to use. Furthermore, the use of multimodal feedback, such as haptics and sound, has proven beneficial for some, but not all, users. This complicates the development of such a virtual environment, as designers are not sure about the implications of the addition of interaction techniques and multimodal feedback. A promising approach for solving this problem lies in the use of adaptation and personalization. By incorporating knowledge of a user's preferences and habits, the user interface should adapt to the current context of use. This could mean that only a subset of all possible interaction techniques is presented to the user. Alternatively, the interaction techniques themselves could be adapted, e.g. by changing the sensitivity or the nature of the feedback. In this paper, we propose a conceptual framework for realizing adaptive personalized interaction in virtual environments. We also discuss how to establish, verify and apply a user model, which forms the first and important step in implementing the proposed conceptual framework. This study results in general and individual user models, which are then verified to benefit users interacting in virtual environments. Furthermore, we conduct an investigation to examine how users react to a specific type of adaptation in virtual environments (i.e. switching between interaction techniques). When an adaptation is integrated in a virtual environment, users positively respond to this adaptation as their performance significantly improve and their level of frustration decrease.
\end{abstract}

Keywords Virtual environments · Adaptation · Framework · User model

\footnotetext{
Johanna Renny Octavia Chris Raymaekers Karin Coninx

Hasselt University - tUL - IBBT

Expertise Centre for Digital Media

Wetenschapspark 2, 3590 Diepenbeek, Belgium

E-mail: \{johanna.octavia,chris.raymaekers,karin.coninx $\} @$ uhasselt.be
} 


\section{Introduction}

Performing complex tasks in virtual environments (VE) requires users to employ highly interactive three-dimensional (3D) interfaces, while keeping the interaction to be natural and intuitive [1]. In medical care, for example, an interactive VE can facilitate surgeons to perform complex operations such as practicing new surgical procedures on simulated patients, but yet still needs to support the surgeons' interaction as naturally as possible. Therefore, employing multimodal interaction becomes essential in the establishment of the VE applications since it makes optimal use of human senses.

The nature of multimodal virtual environments utilizes a wide range of 3D interaction techniques from simple to very complex. When users need to carry out a particular task in such environments, they are confronted with much freedom in deciding which to choose from the various interaction techniques. This situation may possibly introduce additional complexity and cognitive load for users, which in the end might hinder their interaction. We envisage providing adaptive personalized 3D user interfaces as a potential solution to enhance user interaction in virtual environments. Not much research has focused on investigating adaptation and personalization in virtual environments, and in particular, little attention has been spent on adaptation and personalization of $3 \mathrm{D}$ interaction techniques in virtual environments.

The design of adaptive user interfaces is considered as a key figure to improve user interaction with systems by facilitating user performance and helping users deal with complex systems [11]. In this research, we aim to provide adaptive and personalized interaction in virtual environments, which has the ability to adapt to users intelligently, and eventually may increase user performance and enhance user interaction. This vision is disclosed in a conceptual framework for adaptive personalized 3D user interfaces, with the user model as one main building block. This work describes the proposed conceptual framework and the efforts of constructing and employing the user models.

The paper is organized as follows. Section 2 discusses our proposed approach of investigating adaptation in virtual environments, including the survey of related work and the proposition of adaptation types to be investigated in our work. Section 3 introduces the conceptual framework proposed in this work. Section 4 discusses the conduct of user modeling activity through a series of experiments. In Section 5, we describe the first experiment to construct the user model template as a starting point of building user models. Section 6 continues with the description and results of the second experiment that aims to establish individual user models in order to complete building user models in this work. Section 7 concludes the paper.

\section{Adaptation in Virtual Environments}

Due to the complex nature of virtual environments, it becomes important for designers to consider providing adaptation in a virtual environment in order to enhance the interaction of its users. This research prospects the design of adaptive personalized $3 \mathrm{D}$ user interfaces by focusing on adaptation and personalization of 3D interaction techniques in virtual environments. In the context of this paper, we define adaptive and personalized interfaces as interfaces that reflect user characteristics, preferences, needs and abilities to automatically adapt the behavior accordingly using intelligent mechanisms. 


\subsection{Related Work}

Over the past two decades, there has been a quite amount of work in the area of adaptive and personalized user interfaces. Early work included an adaptive personalized interface for a menu-driven application of a telephone-directory system [7], which was shown to be viable and superior to a non-adaptive version. This finding raised the belief that designing systems or interfaces that adapt to users is possible and beneficial.

Several studies have focused on interfaces that adapt to users interacting in the window, icon, menu and pointing (WIMP) applications. Debevc et al. [5] investigated the design and implementation of an adaptive toolbar based on user's frequency of usage of icons. Gajos [6] proposed the idea of automatically personalized user interfaces, mainly in Microsoft Office applications, with the design of SUPPLE and ARNAULD. Rocchi et al. [17] developed an adaptive multimedia mobile guide, which operates on a PDA and presents adaptively selected information to museum visitors based on their expressed interests. Regarding the works on user-adapted systems, a concern has been raised by Chin [4] that stated only a small percentage (about one third) of the works included any type of evaluation and some evaluations were even just pilot or informal studies. More conduct of empirical evaluation with proper design and execution of experiments is encouraged to determine which users are helped or hindered by the user-adapted systems.

Less research has focused on exploring adaptation and personalization of 3D interfaces, most likely due to the phenomenon that interaction in $3 \mathrm{D}$ interfaces is more complex than in WIMP interfaces. Nevertheless, there has been a number of attempts towards it. Celentano et al. [3] discussed adaptivity of interaction in 3D environments by identifying user recurring interaction patterns and using the learned user behavior to shorten user interaction in a virtual world. Wingrave et al. [21] investigated an approach to VE interface design that adapts to users' preferred method of interaction, which later on was introduced as personalized nuance-oriented interaction. Both works share the similar idea of adapting interaction in a virtual environment by learning its user behavior, the first work focused on adaptivity to make user interaction shorter and the latter focused on adaptivity to create a more preferred interaction technique.

Our work is inspired by Wingrave et al. [21], where the designer learns the user's method of interaction and personalize the interaction technique to behave more like what the user wants. We carry on the same notion towards adaptive 3D interaction techniques with proposing other different types of adaptation, which will be discussed later on in this section. Particularly in this paper, we focus on investigating a specific type of adaptation, which is switching between different interaction techniques to offer the most suitable interaction technique for a user in a certain situation.

\subsection{Towards Adaptive 3D Interaction Techniques}

According to Bowman et al. [2], interaction techniques are defined as methods that allow a user to accomplish a given task via the user interface. Three-dimensional (3D) interaction techniques can be defined as methods used to complete the users tasks such as selection, manipulation, and navigation in a 3D spatial context. For example, when performing a $3 \mathrm{D}$ object selection task in a virtual environment, a user can employ selection techniques based on a virtual hand metaphor such as the Go-Go technique [15] 
or a virtual pointer metaphor such as the Ray-casting technique [16]. A complete classification of 3D interaction techniques can be found in [2].

Much research into three-dimensional (3D) interaction techniques has been conducted over the past years. However, the usability of many interactive VE applications has not yet achieved a desirable level. Bowman et al. [1] proposed considering userspecificity in the design of 3D user interfaces to improve the usability of 3D interaction techniques. It has been mentioned earlier that we intend to enhance user interaction in virtual environments through integration of adaptation into 3D interaction techniques. Adaptation in virtual environments has quite a broad scope since designers and developers can make efforts to support as many types of adaptation as they can think of. However, to realize all in one system is quite cumbersome. In this research, we would like to focus on investigating three types of adaptation: (I) switching between interaction techniques, (II) adapting the interaction technique itself, and (III) enhancing the interaction technique with modalities.

\subsubsection{Switching between Interaction Techniques}

When performing tasks in virtual environments, users may encounter different situations (e.g. environment condition of the virtual world, position of the user) that might influence the performance of executing the interaction technique differently as well. There is no single technique that works best in all situations. For instance when selecting an object in a dense environment, users may prefer a technique using a virtual hand metaphor over a technique using a virtual pointer metaphor, or vice versa. This preference might deviate when selecting an object in a sparse environment. We are interested to investigate the possibility of enabling users to switch between different interaction techniques while performing the same task, as a result of adapting to different environment conditions. This type of adaptation results in the action of offering the most suitable interaction technique for a user in a certain situation.

\subsubsection{Adapting the Interaction Technique itself}

Every user has a certain physical ability level that may have effect on performing an interaction technique in virtual environments. For example, a user may have a lot of hand tremor that decreases his efficiency and accuracy in accomplishing an object selection task using a particular selection technique. We could change certain parameters (e.g. viscosity, force strength) of a task as an adaptation to the user's specific attributes (e.g. degree of tremor). Therefore, we are also intrigued to investigate the possibility of adapting the interaction technique itself by adjusting its sensitivity according to the user's need. This type of adaptation is basically adjusting the parameters of the interaction technique to control how the user should perform it.

\subsubsection{Enhancing the Interaction Technique with Modalities}

Besides adjusting the parameters, we can also adapt the interaction technique by enhancing it with the use of multimodal feedback such as visual, audio or force feedback. Particular users may perform a selection task better when the selection technique is complemented by haptics (force feedback) as such that they can have more control on how the technique behaves. In our previous work $[18,19]$, we have already experimented with this last type of adaptation. 


\section{Conceptual Framework}

We are aware of the importance of enhancing user interaction in virtual environments through the establishment of adaptive personalized 3D user interfaces. Our goal is to support adaptation and personalization in order to assist users when interacting in virtual environments. This includes determining which interaction technique is suitable for a user in a certain situation (e.g. by only providing a limited number of interaction techniques) and adapting the interaction techniques themselves (e.g. by adjusting the sensitivity of the interaction). The adaptation should occur not only according to the users' characteristics, such as their preferences and abilities, but also to the actions they performed in the past and the task they are currently executing. To achieve this, we propose our conceptual framework as depicted in Figure 1.

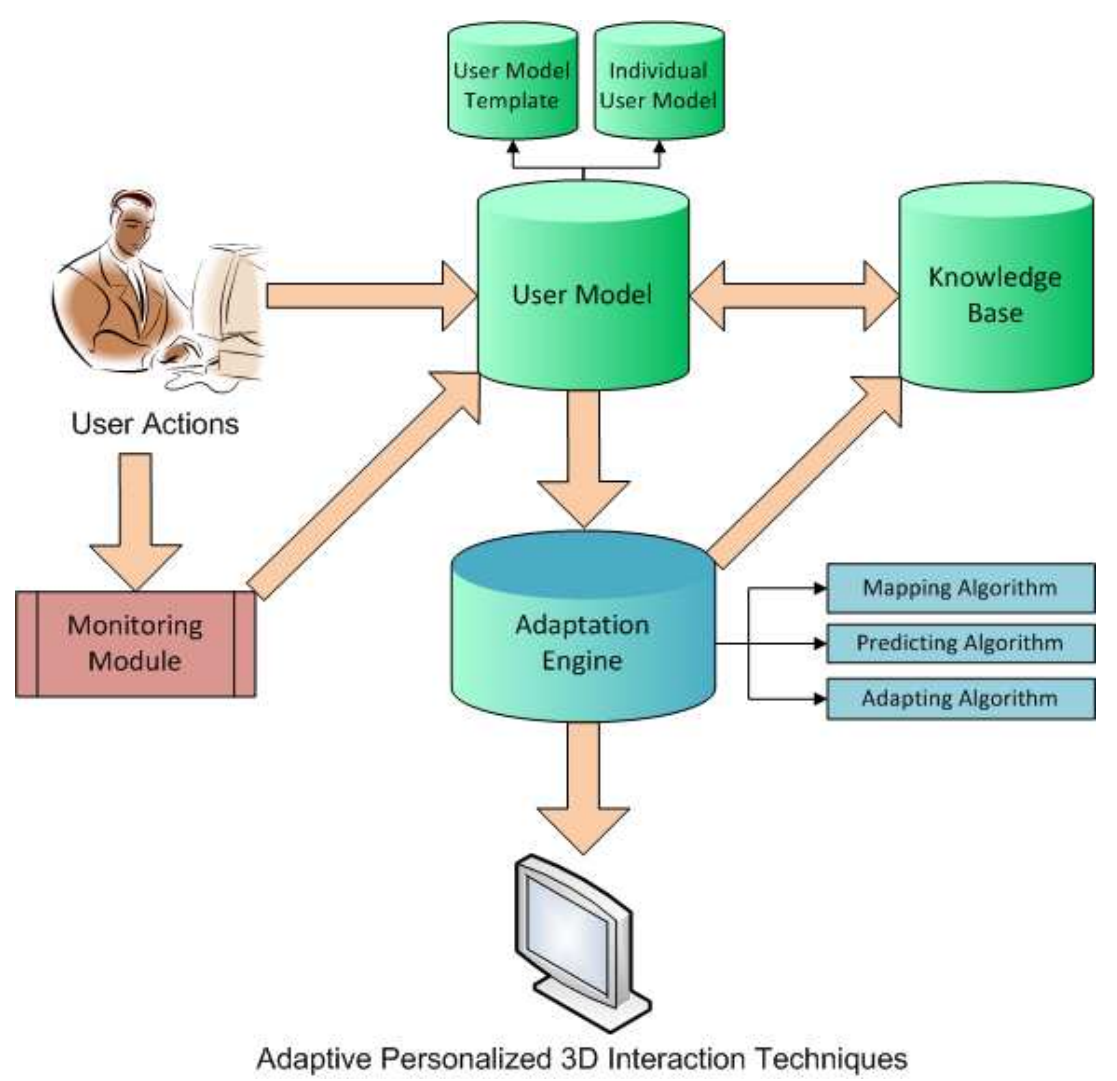

Fig. 1 Conceptual framework for adaptive personalized 3D user interfaces

The main goal of this framework is to come up with 3D interaction techniques that are appreciated by users in a particular context of use through adaptation and personalization. With the framework, we intend to gather information and build knowledge about users on their working methods, performances, preferences and abilities when interacting in virtual environments. This knowledge will later on be used to assess the adaptation and personalization of interaction techniques, either deciding which partic- 
ular interaction technique should be offered or regulating how an interaction technique should behave, depending on the performance and preference of users.

When interacting in virtual environments, users carry out certain actions. The recurring interaction patterns are identified and delivered into the user model. User actions are also recognized and monitored as triggers for adaptation in the monitoring module. When it identifies new patterns, the user model will be updated. When assessing the trigger, the user model closely collaborates with the knowledge base, which contains factual information about the past interaction and adaptation. This collaboration provides information to the adaptation engine, where the adaptation and personalization take place with the help of intelligent algorithms. As a result, adaptive personalized 3D user interfaces can be realized.

To support the role of the adaptation engine, three intelligent algorithms are foreseen, namely the mapping algorithm, the predicting algorithm and the adapting algorithm. The mapping algorithm can help to selectively offer interaction techniques or metaphors to users depending on the actual context of use. The predicting algorithm can help to enable or disable interaction techniques based on the prediction made according to the semantic of objects and the history of prior used interaction techniques. The adapting algorithm can help to determine whether it is desirable to adapt the interaction technique or enhance it with other modalities such as audio or force feedback.

In this work, we focus on the establishment of one component proposed in the framework, namely the user model. We realize that the conceptual framework consists of several building blocks that need to be further investigated.

\subsection{User Model}

To accommodate user diversity, building user models becomes crucial to provide adaptation and personalization for users. User models contain information and assumptions about users that play an important role in the adaptation process of user interfaces to the needs of different users. The user model is acquired through user modeling activity, which attempts to gather users' interaction patterns, preferences, interests and characteristics.

Jameson and Wittig [9] proposed a user modeling approach that learns two types of user models, namely general user models and individual user models. A general user model is learned from all observations of a sample of users and can be applied to all users in general. An individual user model is learned completely from observing one particular user and later can be adapted during the interaction. Another user modeling method is the stereotype approach discussed by Kobsa [10], which identifies user groups and generalizes their interaction behaviors into patterns to construct group user models. Not much attention has been spent on the effort of establishing user models for the purpose of adaptation and personalization in virtual environments.

In this work, the user modeling activity is conducted through a series of experiments that apply existing user modeling approaches $[9,10]$. As can be seen in the framework, we employ a user model template combined with individual user models. The user model template is developed by establishing general and group user models. This template is used as a starting point in adaptation process for users that do not have any prior interaction history. The user modeling activity performed to construct the user model in this work is further discussed in Section 4. 
General user models are advantageous to people using a virtual environment for the first time to benefit instantly from adaptation and personalization. Group user models are employed because we believe users interacting in virtual environments can be classified into different user groups based on different user characteristics such as experience level, gender or age. However, proposing the adaptation only based on the user model template is insufficient because users remain individuals that vary so much from each other. Therefore, individual user models are employed in parallel with the user model template in such a way that an individual user model refines the user model template.

There are various levels of information provided in the user model. The general user model provides the most basic information that can be used for adaptation to all users. The group user model gives more specialized information to be applied to a group of users, while the individual user model delivers the most detailed information about one particular user. When the information is conflicting between the levels, the most specific information (e.g. individual user model) takes priority over more general information (e.g. group user model).

\section{User Modeling through Experiments}

We initiate the implementation of the conceptual framework by constructing the user models. As proposed in Section 3.1, we will build a user model template (consisting of general and group user models) and individual user models. We intend to achieve this by conducting user modeling activity through carrying out two subsequent experiments. The objective of the first experiment is to construct the user model template, while the second experiment aims to establish the individual user model. Both experiments are designed based on the same context and used the same setup described as follows.

\subsection{Context of the Experiments}

In this work, we focused on investigating user interaction when performing selection tasks in a virtual environment with controlled variables, such as the density of objects, target distance and occlusion. Object selection is a fundamental task in virtual environments and any interactive 3D user interfaces must support performing this task. Vanacken et al. [20] evaluated several selection techniques for dense and occluded virtual environments and found that the bubble cursor technique and the depth ray technique perform better than other techniques. Therefore, we chose to base our experiment on these two selection techniques as shown in Figure 2.

The bubble cursor (Figure 2(a)) is based on the hand extension metaphor, which resembles the action of reaching out one's hand to the target object to be selected. The hand is represented by a crosshair or 3D axis, which is rendered inside the bubble cursor and corresponds to the center of the cursor. Around the 3D axis, an invisible sphere resizes dynamically as such that only the closest object is captured. The captured object will be highlighted yellow and can be selected. Objects in close proximity to the bubble cursor become semi-transparent, so that occluded targets may become visible when approached.

The depth ray (Figure 2(b)) is based on the ray casting metaphor, which resembles the action of pointing one's hand at the target object to be selected. The hand is 


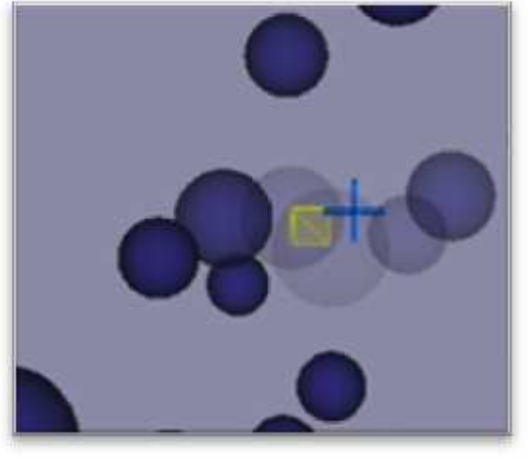

(a) The bubble cursor

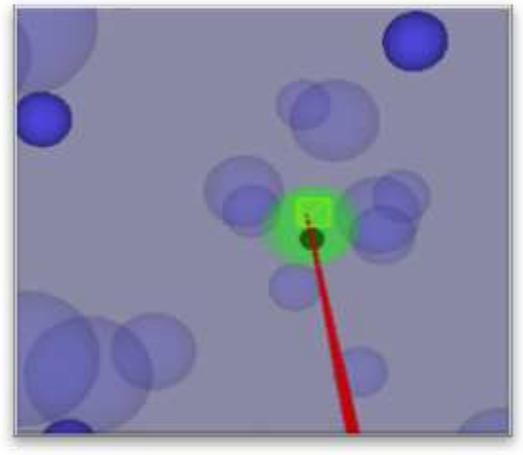

(b) The depth ray

Fig. 2 The selection techniques used

represented by a ray, which is rendered as a thin red cylinder with an apex of $1^{\circ}$. Objects that intersect with the ray will be highlighted green. Along the ray, there is a depth marker (rendered as a black sphere) that can be controlled by moving the hand forward or backward. The object intersected by the ray cursor, which is closest to the depth marker, can be selected. Objects in close proximity to the ray become semi-transparent, to help making occluded targets visible.

\subsection{Setup of the Experiments}

The experiments were conducted using the same setup with regard to the experiment environment and apparatus. Prior to the experiment, we generated scenes of the environment by manipulating the environment density, target distance and target occlusion. As shown in Figure 3, the scene consists of a start target (white sphere), a goal target (red sphere), and 45 distractor targets (blue spheres). In each scene, the start target is always centrally positioned at the front of the display, while the goal and distractor targets are randomly positioned. Each trial begins with a fade-in of the scene. First only the goal target would be visible, then the distractor targets would fade in over a duration of 2 seconds, followed by the appearance of the start target. This would give participants an idea of the goal target's location for the occluded conditions beforehand.

The experiment apparatus used in the experiments is illustrated in Figure 4. A Polhemus Fastrak 6 DOF magnetic tracker was used as input device, built in a handheld case as shown in Figure 4(a). The device was equipped with a single button to indicate selection. The tracker was updated at $120 \mathrm{~Hz}$ with precision of less than $1 \mathrm{~mm}$. The input device controlled each of the two cursors with an absolute one-to-one mapping. The display used was a polarization projection screen $(2.4 \mathrm{~m} \times 1.8 \mathrm{~m})$ with passive stereo using two DLP projectors as shown in Figure 4(b). During the experiment, participants stood at the designated position which is about $1 \mathrm{~m}$ in front of the center of the projection screen. 


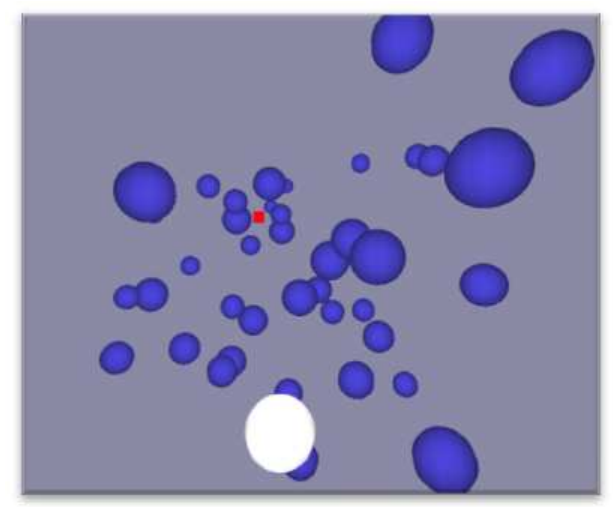

Fig. 3 The experiment environment

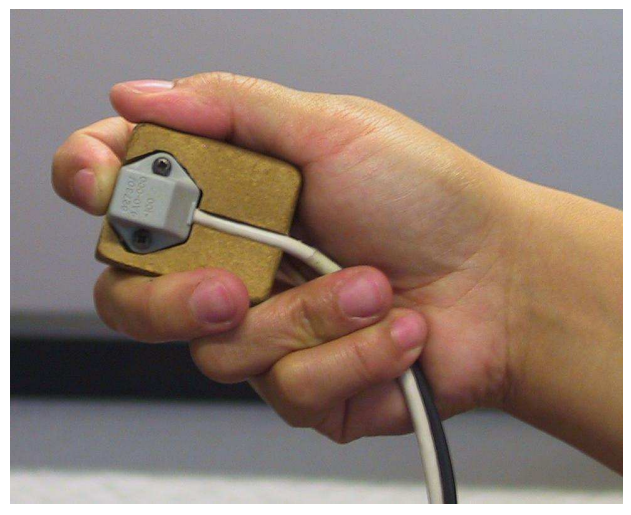

(a) The magnetic tracker

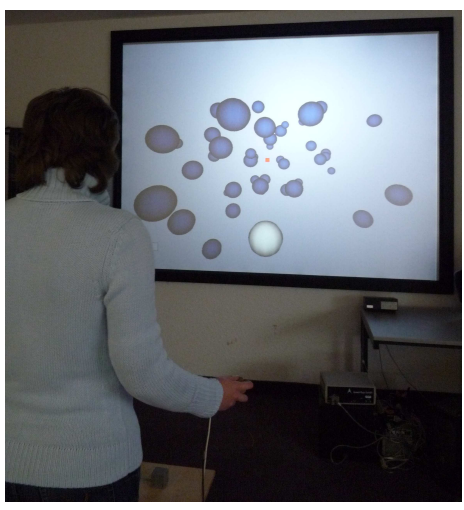

(b) The projection screen

Fig. 4 The experiment apparatus

\section{Experiment 1: Establishment of User Model Template}

To begin constructing the user model template, we conducted the first experiment on $3 \mathrm{D}$ target acquisition task in virtual environments. As previously mentioned in Section 3.1, the user model template comprises the general user model and group user models. The general user model is constructed based on general information about all users, therefore it provides the most basic information that can be used for adaptation for all users. Due to diversity of user groups, group user models are employed based on different user characteristics (e.g. experience level, gender or age). The group user model gives more specialized information than the general user model, only to be applied to a group of users.

\subsection{Participants}

Four user groups differing in experience level and gender, namely novice females, novice males, experienced females and experienced males, participated in this experiment [14]. 
We define novices as ones who do not have any experience at all interacting in a virtual environment such as playing 3D video games or using softwares. Sixteen participants between 18 and 31 years old were recruited for this experiment, which lasted for about 45 minutes. None of the novice participants had experience with virtual environments. All experienced participants were moderately experienced with virtual environments, reporting that they played $3 \mathrm{D}$ video games or used $3 \mathrm{D}$ softwares quite often at least 3 times a week. All participants were screened using the Stereo Fly Test SO-001 ${ }^{1}$ to check their stereoscopic depth perception. All participants were right-handed and used their right hand to operate the device. In the experiment, participants were asked to select a goal target randomly positioned among distractor targets, as fast as possible while minimizing errors.

\subsection{Results}

In the experiment, we found that experience level has effects on users' performance for 3D target acquisition task in a virtual environment, while gender not. Experienced users perform significantly better than novices, while males and females do not perform significantly different. Even so, it was also found that there is no difference between the user groups with regard to the selection technique itself. Prior to the experiment, we intended to build a user model template consisting of general and group user models. However, the findings of this experiment were deviated slightly. We expected to be able to construct group user models based on the differences between those user groups with respect to their performance and preference for interaction technique. Due to the fact that no trends were found, group user models were unsuccessful to be constructed. Nevertheless, we were aware that besides experience level and gender, there are other user characteristics that can be taken into account, such as age, physical characteristics, physical and cognitive abilities.

We proceeded with another element of the user model template which is the general user model. As shown in Figure 5, we constructed the general user model based on the performance and preference of participants as a whole. Table 1 shows the general user model in summary. This provides information on the appropriate selection technique for executing 3D target acquisition task in a virtual environment, for all users in general.

Table 1 General user model (summary)

\begin{tabular}{ll}
\hline Environment condition & Interaction technique \\
\hline Occluded-Dense-Near (ODN) & Bubble Cursor \\
Occluded-Dense-Distant (ODD) & Bubble Cursor \\
Occluded-Sparse-Near (OSN) & Bubble Cursor \\
Occluded-Sparse-Distant (OSD) & Bubble Cursor \\
Visible-Dense-Near (VDN) & Bubble Cursor \\
Visible-Dense-Distant (VDD) & Depth Ray \\
Visible-Sparse-Near (VSN) & Bubble Cursor \\
Visible-Sparse-Distant (VSD) & Depth Ray \\
\hline
\end{tabular}

This general user model can be beneficial for first-time users interacting in a virtual environment who do not have any interaction history at all. For instance, users can

1 http://www.stereooptical.com/html/stereo-test.html 


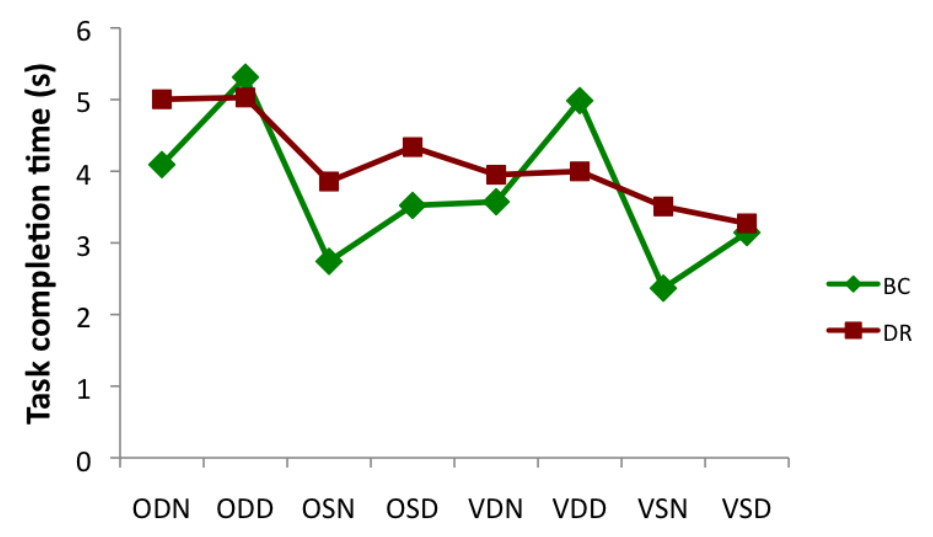

Environment condition

(a) Performance-based

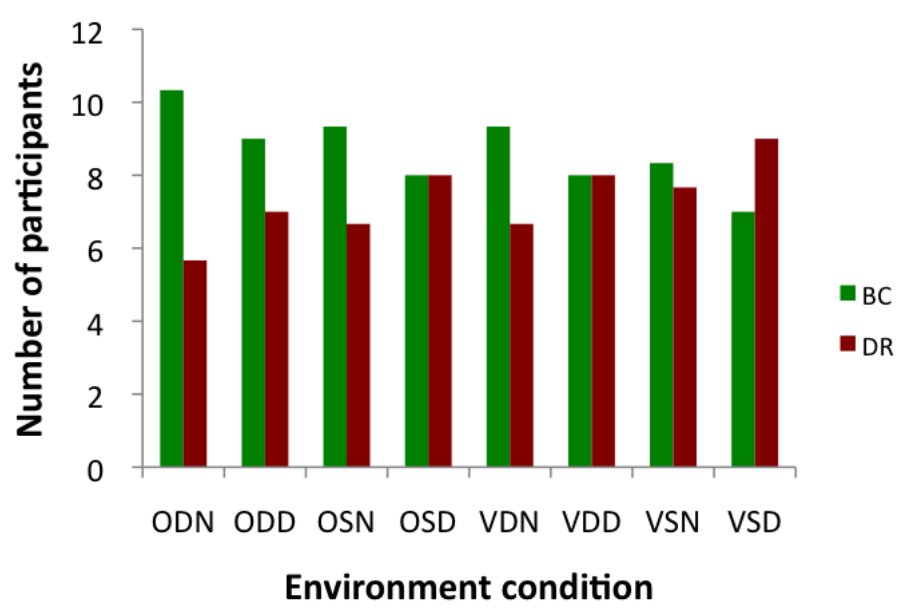

(b) Preference-based

Fig. 5 General user model based on performance and preference of all users

be offered to use the depth ray technique when they start interacting in a virtual environment with dense objects, visible and distant target. When the environment switches to the one with sparse objects, occluded and distant target, they can be offered the bubble cursor technique. By applying the general user model, we expect that the users' interaction may be optimal, even though they are still novice to virtual environments.

However, the general user model presented in this section stills need to be verified, whether our presumption is true or not that the model is favorable to enhance first-time users' interaction in a virtual environment. This effort of verification will be discussed in the next section. For a more detailed description of the findings, design and procedure of this first experiment, readers are referred to [14]. 


\section{Experiment 2: Establishment of Individual User Models}

Aligned with the user model template, we also employ individual user models. The individual user model serves an important role in the adaptation process of user interfaces for every particular user. Especially since we observed in the first experiment that every user has his/her own interaction pattern that differs from person to person. To construct an individual user model, we need to build knowledge on a user's performance and preference by analyzing each user individually. This user model requires prior traces of interaction of one user, and will be continuously adjusted during the interaction itself. Compared to a general user model that is static, an individual user model is dynamic.

We conducted the second experiment to construct the individual user model in order to complete the establishment of user models for this study. Furthermore, another goal of the experiment was to verify the general user model illustrated in Section 5 . Through this experiment, we would also like to investigate how users react to adaptation of interaction technique based on the result of combining the general and individual user models.

\subsection{Participants}

In this experiment, sixteen participants between 24 and 32 years old were recruited and divided into two user groups, eight first-timers and eight non first-timers. We define first timers as ones who never experience interacting in our particular virtual environment designed for the experiment. We asked people who participated in the previous experiment as non first-timers in this experiment. All participants were righthanded and screened using the Stereo Fly Test SO-001.

\subsection{Physiological data collection}

One objective of the experiment was to investigate the user's reaction to adaptation. For this purpose, we gathered physiological data from participants in order to assess user frustration with regard to adaptation. Therefore, in addition to the experiment apparatus mentioned in Section 4.2, we also utilized the ProComp Infiniti hardware ${ }^{2}$ and Biograph software, a device for real-time computerized biofeedback and physiological data acquisition, as shown in Figure 6(a).

In this experiment, we used the ProComp Infiniti device to collect participants' two kinds of physiological data, namely skin conductance (Galvanic skin response - GSR) and muscle activity (Electromyography - EMG). We chose to collect these physiological measures based on previous work that made use of psychophysiological techniques (e.g. to measure user frustration [8], to investigate user experience [12] and to evaluate mental workload [13]). Figure 6(b) shows how the device and two sensors for measuring skin conductance and electromyography were attached to a participant during the experiment.

2 http://www.thoughttechnology.com 


\subsubsection{Skin conductance}

Galvanic skin response (GSR), or widely known as skin conductance, is a measure of the ability of skin to conduct electricity. Changes in skin conductance measures can be seen as a reflection of changes in emotional arousal such as fear or frustration. For example, as a person becomes more or less stressed, the skin conductance increases or decreases proportionally. We measured skin conductance using the Skin Conductance sensor (SC-Flex/Pro) which is placed around the index finger and the little finger of the same hand. Skin conductance is measured in micro-Siemens $(\mu S)$. Normally in a relaxed state, the skin conductance is around $2 \mu S$, but this can vary considerably with skin type and other environmental factors. It is more important to examine the alteration of skin conductance measures from the baseline, whether it is increasing or decreasing.

\subsubsection{Electromyography}

Electromyography (EMG) is a measure of muscle activity by detecting electrical impulses generated when muscle fibers contract. When measured on the forehead or jaw, EMG provides an indicator of tension due to the wrinkling of forehead or clenching of jaw. Electromyography is a precise method to measure changes in facial expression that reflect the individuals current emotional state. In this experiment, the facial muscle activity was measured by placing the Surface Electromyography sensor (MyoScan-Pro) on the forehead of participants. The measurement unit of electromyography is microvolts $(\mu V)$. Normal resting value of electromyography is usually around 3 to $5 \mu V$. Since this value also vary greatly, it is more necessary to look at the changes in amplitude, which are directly proportional to the muscle's activity.

\subsection{Procedure}

The experiment consisted of two sessions, which were conducted over the course of two days. The first session was conducted to build the individual user model and lasted for about 45 minutes. In this session, participants were asked to perform a $3 \mathrm{D}$ target acquisition task using the two selection techniques. Just as in the first experiment, they had to select a goal target randomly positioned among distractor targets, as fast as possible while minimizing errors. Participants were also asked to state their preferred technique for each environment condition. As shown in Figure 7(a), first they were asked to choose which technique they prefer to use and then rate their preference.

The second session aimed for testing the general user model and inquiring insights into the user's reaction to adaptation. The individual user model resulted from the first session was combined with the user model template, then this action informed the system to decide further concerning the adaptation of interaction technique. During this session, participants were simply asked to perform the 3D target acquisition task with the proposed technique (result of adaptation based on general and individual user model), as depicted in Figure 7(b). This session lasted for about 20 minutes. At the end of both sessions, participants were given a questionnaire to gather their subjective responses regarding user's mood, user's frustration and task difficulty. 

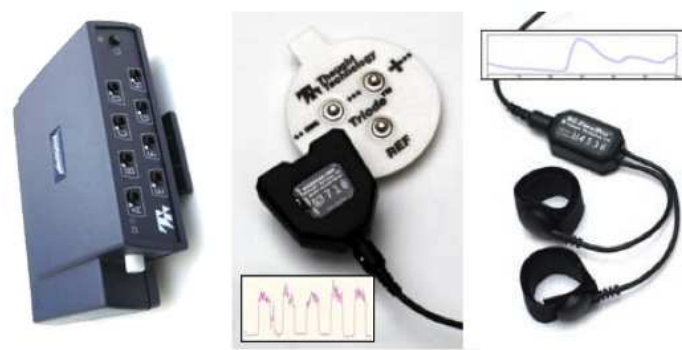

(a) ProComp Infiniti encoder, Surface Electromyography sensor, Skin Conductance sensor

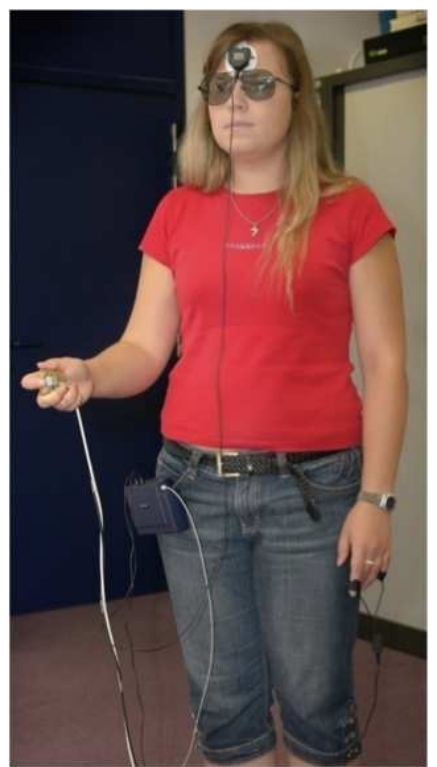

(b) One participant using ProComp Infiniti and sensors

Fig. 6 Gathering physiological data in the experiment

\subsection{Results}

\subsubsection{Individual User Models}

In the first session of this experiment, we collected performance and preference data of every participant. Based on the knowledge built by analyzing each participant individually, we were able to determine their individual user models. The individual user model gives information about the most suitable selection technique for executing 3D target acquisition task in every environment condition of a virtual environment, for one particular user based on his/her objective performance and subjective preference of interaction technique. Table 2 shows examples of the individual user model for a first-timer participant and a non first-timer participant. As can be observed, both individual user models are quite different. This diversity is also shown when we compared participants within each user group. However, we also noticed a tendency for some participants to have an individual user model which is uniform across all conditions. Some of the first-timer participants were best with bubble cursor for all conditions, while some of the non first-timer participants were best with depth ray for all conditions.

Based on their individual user models, we proposed the selection technique for each environment condition as a result of adaptation. During the second session of the experiment, this proposition of interaction technique were presented to the participants without them being aware of it. We just asked them to execute the 3D target acquisition task with the technique shown in the projection screen. At the beginning, some participants found it quite confusing because the system keeps on switching between interaction technique automatically. But after a while, they became used to it and did 


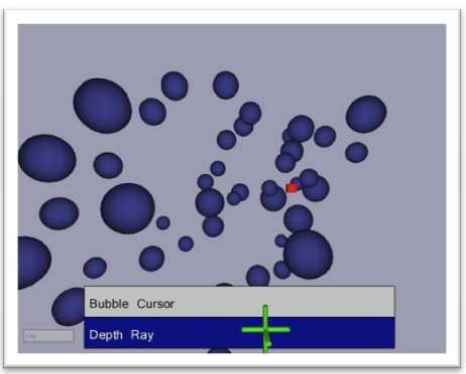

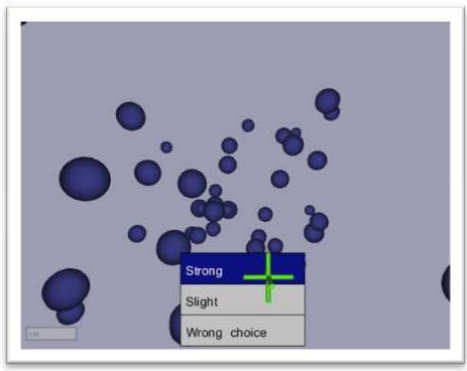

(a) Preference elicitation

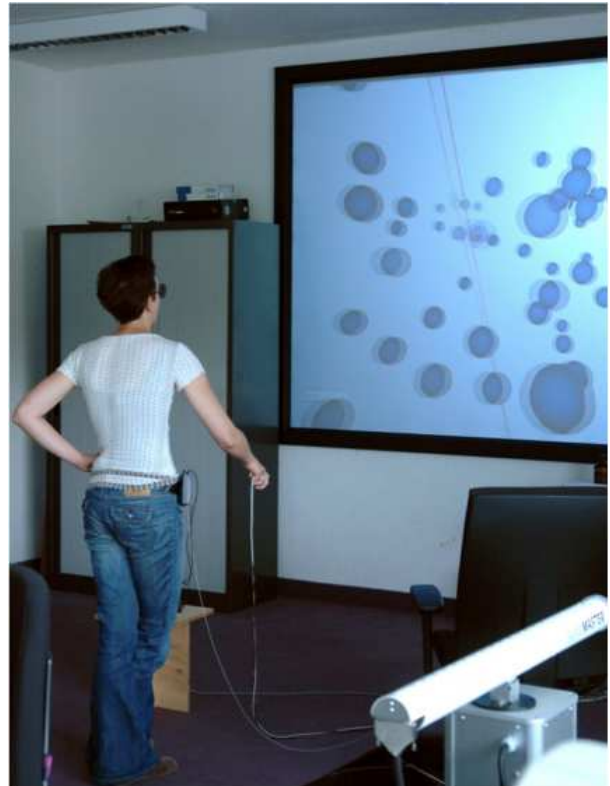

(b) One participant carrying out the experiment

Fig. 7 Overview of the second experiment sessions

Table 2 Examples of individual user models

\begin{tabular}{lll}
\hline & \multicolumn{2}{c}{ Interaction technique } \\
\cline { 2 - 3 } Environment condition & Non first-timer & First-timer \\
\hline Occluded-Dense-Near (ODN) & Bubble Cursor & Depth Ray \\
Occluded-Dense-Distant (ODD) & Bubble Cursor & Bubble Cursor \\
Occluded-Sparse-Near (OSN) & Depth Ray & Depth Ray \\
Occluded-Sparse-Distant (OSD) & Bubble Cursor & Bubble Cursor \\
Visible-Dense-Near (VDN) & Bubble Cursor & Depth Ray \\
Visible-Dense-Distant (VDD) & Bubble Cursor & Depth Ray \\
Visible-Sparse-Near (VSN) & Depth Ray & Bubble Cursor \\
Visible-Sparse-Distant (VSD) & Bubble Cursor & Depth Ray \\
\hline
\end{tabular}

not feel disturbed anymore. Some participants also became aware of the adaptation implemented and felt positive about it. These phenomena can be observed from several remarks from participants as follows.

\footnotetext{
"At first, it was a little confusing for me when the system switched the technique automatically because suddenly I had to adapt. But only for the first few times..."

"Mixing the two interaction techniques does not really bother, I'm not disturbed by changing selection techniques."

"I didn't have to use the depth ray anymore! The bubble cursor was a lot easier to use."

"I always seemed to have the right tool for the job!"
} 


\subsubsection{Verification of General User Model}

As part of the second session of the experiment, we also tested the practice of the general user model presented in Section 5. We hypothesized that employing general user model will increase user performance and decrease user frustration compared to the condition before employing any user model. Our second hypothesis was that employing an individual user model will increase user performance and decrease user frustration compared to the condition of employing the general user model.

We analyzed three conditions of adaptation in this experiment: (I) no adaptation, (II) adaptation with the individual user model, and (III) adaptation with the general user model. One-way analysis of variance (ANOVA) showed a significant main effect of Condition on Task completion time $\left(F_{2,45}=5.93, \mathrm{p}<0.005\right)$. This indicates a significant difference between the three conditions. Average task completion times were $8.76 \mathrm{~s}$ for condition of no adaptation, $5.19 \mathrm{~s}$ for condition of adaptation with the individual user model, and $6.13 \mathrm{~s}$ for condition of adaptation with the general user model. Post hoc comparisons showed that both conditions of adaptation with the individual user model and the general user model were significantly faster than the condition of no adaptation $(\mathrm{p}<0.05)$. However, the condition of adaptation with the individual user model was not significantly faster than the condition of adaptation with the general user model $(\mathrm{p}=0.66)$.

Beside task completion time, we also compared the three conditions based on the physiological data (electromyography and skin conductance value). As mentioned in Section 6.2.1 and Section 6.2.2, it is more worthwhile to investigate the alteration of skin conductance and electromyography measures from the baseline since the values may vary greatly from person to person. Therefore for each condition, we calculated the average of increments of the skin conductance and electromyography values from the baseline. We again conducted a one-way analysis of variance (ANOVA) which showed no significant main effect of Condition on Electromyography increment $\left(F_{2,45}=0.05\right.$, $\mathrm{p}=0.96$ ). Based on the electromyography measures, there was no indication of difference between the three conditions. Generally we can say that in every condition, users did not have as much facial muscle activity as expected when they are tensed. On the other hand, it showed a significant main effect of Condition on Skin conductance increment $\left(F_{2,45}=5.99, \mathrm{p}<0.005\right)$. So when we only analyze based on the skin conductance measures, we can say that this finding also indicates a significant difference between the three conditions. Average skin conductance increment were $1.74 \mu S$ for condition of no adaptation, $0.52 \mu S$ for condition of adaptation with the individual user model, and $0.83 \mu S$ for condition of adaptation with the general user model. Post hoc comparisons showed that both conditions of adaptation with the individual user model and the general user model were significantly lower than the condition of no adaptation $(\mathrm{p}<0.05)$. However, the condition of adaptation with the individual user model was not significantly lower than the condition of adaptation with the general user model $(\mathrm{p}=0.68)$.

Based on our analysis, we discovered that employing user models (either general or individual) increases user performance and decreases user frustration. In the experiment, participants performed faster when user models were applied to result in adaptation and they were also less frustrated which was shown by the decrease in their skin conductance values. We also found out that providing adaptation based on individual user model is better than general user model, although not significantly better. In another way, we can also say that basing adaptation only on general user model may 
work for most users. Therefore, we can claim that the general user model is verified to be beneficial for first-time users interacting in a virtual environment.

\subsubsection{First-timers vs. Non first-timers}

We contrasted non first-timer and first-timer participants to observe the difference between these user groups. Two-way analysis of variance (ANOVA) showed a significant main effect of Experience on Task completion time $\left(F_{1,42}=8.02, \mathrm{p}<0.005\right)$ and also main effect of Condition on Task completion time $\left(F_{2,42}=7.09, \mathrm{p}<0.005\right)$. This indicates a significant difference between the two user groups. However, we found no significant interaction effect between Experience and Condition, which showed that first timers perform significantly slower than non first-timers regardless of the condition. Without adaptation, average task completion times were $10.98 \mathrm{~s}$ for first-timers and $6.54 \mathrm{~s}$ for non first-timers. For adaptation with the individual user model, average task completion times were $5.59 \mathrm{~s}$ for first-timers and $4.81 \mathrm{~s}$ for non first-timers. For adaptation with the general user model, average task completion times were $6.92 \mathrm{~s}$ for first-timers and $5.53 \mathrm{~s}$ for non first-timers.

We also looked at the trends of skin conductance value of these two user groups. Figure 8 and Figure 9 illustrate the skin conductance values of a first-timer and a non first-timer participant for the session without adaptation and with adaptation. As we can see, there is a pronounced decline in the skin conductance value after the integration of adaptation, both for the first-timer and non first-timer participant.

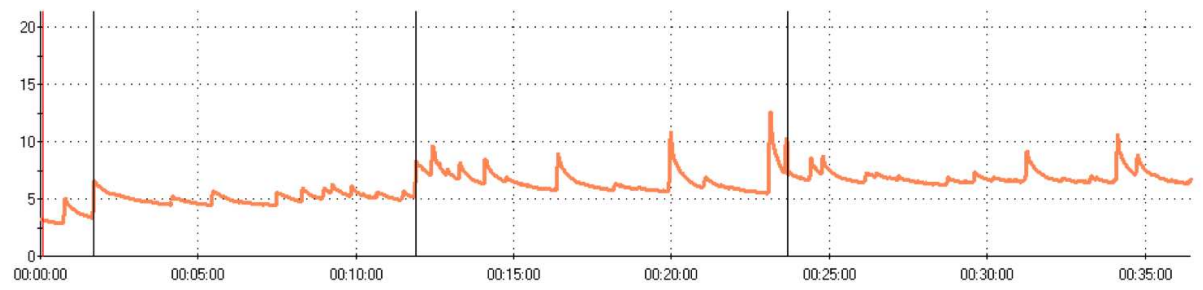

(a) Without adaptation

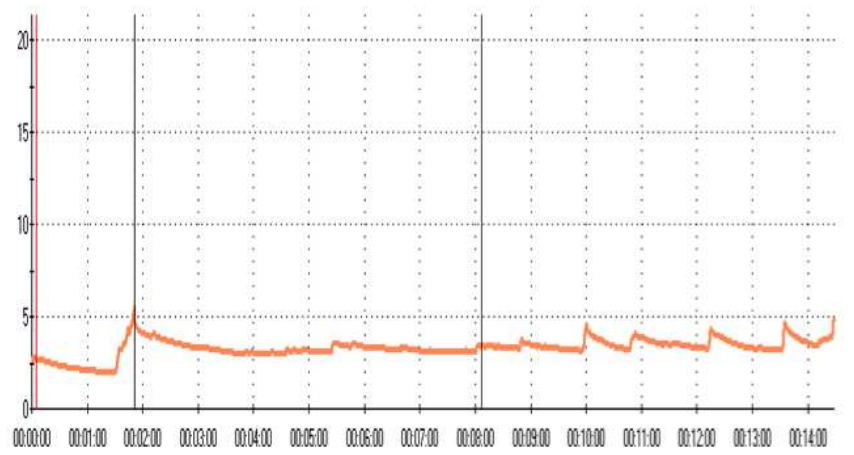

(b) With adaptation

Fig. 8 Skin conductance values of a first-timer participant $(\mu S)$ 


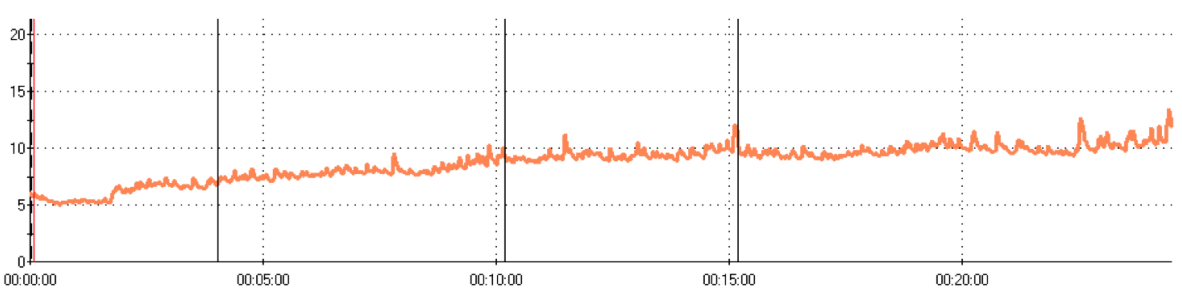

(a) Without adaptation

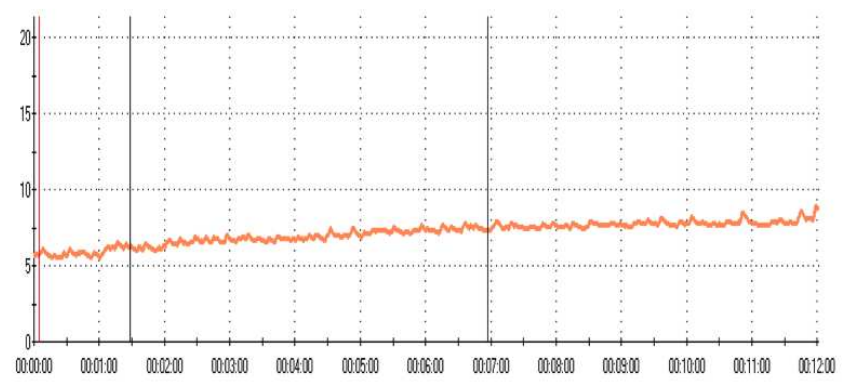

(b) With adaptation

Fig. 9 Skin conductance values of a non first-timer participant $(\mu S)$

\subsubsection{User's Reaction to Adaptation}

As briefly discussed in Section 6.4.1 and 6.4.2, we have revealed the findings that participants responded well to the adaptation of interaction technique implemented in the experiment. First, we observed positive reactions from the comments that some participants passed on throughout the experiment (see Section 6.4.1 for more details). The remarks gave us an impression that the adaptation was acceptable and they felt good about the proposed techniques from the system, which fit more to their preference. And secondly, based on the statistical analysis on the task completion time and physiological data, we found that participants perform significantly better and experience less frustration when adaptation was introduced in the system.

To investigate further on users' reaction to adaptation, we gathered subjective responses from participants through a questionnaire at the end of the experiment sessions. We asked them to indicate their level of mood, frustration and the level of task difficulty, on a 7 point scale rating (e.g. 1 not frustrated at all to 7 very frustrated). We conducted a linear regression analysis to observe the relationship between the objective data (i.e. task completion time) and the subjective data (i.e. ratings on mood, frustration, and task difficulty). We found that there is a significant positive relationship between Task completion time and Rating on frustration $\left(r_{s}=0.39, \mathrm{p}<0.05\right)$. This implies that the rating on frustration was strongly related to the variation in data of task completion time. Furthermore, a chi-square analysis on the subjective ratings showed a significant effect for Rating on frustration $\left(\chi^{2}(6)=12.78, \mathrm{p}<0.05\right)$ and Rating on task difficulty $\left(\chi^{2}(4)=17.14, \mathrm{p}<0.005\right)$. This finding shows that participants undergo less frustration and perceive performing the task less difficult when adaptation is incorporated. 


\section{Conclusions}

In this paper, we have discussed our idea of providing adaptation in virtual environments in the belief that adapting the user interface enhances user interaction in the long run. We disseminated this vision in a conceptual framework for realizing adaptation and personalization of interaction techniques in virtual environments. As a first step in implementing the proposed framework, we also have described our effort to build the user model, both the user model template and individual user models, as an important building block of the framework. Two experiments were conducted to achieve the establishment, verification and application of the user model in this research. The first experiment resulted in a user model template; which then in the second experiment the general user model was verified to benefit users interacting in a virtual environment. The second experiment led to the establishment of individual user models.

Based on the constructed user model, we also implemented the adaptation of switching between interaction techniques in virtual environments and investigated user's reaction to adaptation. We found a positive response from users concerning the adaptation of interaction technique implemented in the second experiment. Users perform significantly better and experience less frustration when adaptation is incorporated during their interaction in virtual environments. Subjectively, users also showed that adaptation was satisfactory since they were favorable to the interaction technique proposed by the system as a result of adapting to their preference and performance.

Following the investigation of user models, we also realize that further studies of the various building blocks of the framework still need to be conducted. Performing similar studies that consider other 3D interaction techniques such as navigation or manipulation may be compelling to explore more possibilities for adaptation and personalization in virtual environments.

Acknowledgements The authors would like to thank Lode Vanacken for his assistance in the implementation work for the experiment. We also thank the participants for their valuable contributions in the experiment.

\section{References}

1. Doug A. Bowman, Jian Chen, Chadwick A. Wingrave, John Lucas, Andrew Ray, Nicholas F. Polys, Qing Li, Yonca Haciahmetoglu, Ji-Sun Kim, Seonho Kim, Robert Boehringer, and Tao Ni. New directions in 3d user interfaces. Int. J. Virtual Reality, $5(2): 3-14,2006$.

2. Doug A. Bowman, Ernst Kruijff, Joseph J. LaViola, and Ivan Poupyrev. 3D User Interfaces, Theory and Practice. Addison-Wesley, 2005.

3. A. Celentano, M. Nodari, and F. Pittarello. Adaptive interaction in web3d virtual worlds. In Proceedings of the 9th 3D Web, pages 41-50, 2004.

4. David N. Chin. Empirical evaluation of user models and user-adapted systems. User Modeling and User-Adapted Interaction, 11(1-2):181-194, 2001.

5. Matjaz Debevc, Beth Meyer, Dali Donlagic, and Rajko Svecko. Design and evaluation of an adaptive icon toolbar. User Model. User-Adapt. Interact., 6(1):1-21, 1996.

6. Krzysztof Z. Gajos. Automatically Generating Personalized User Interfaces. PhD thesis, University of Washington, 2008.

7. S. Greenberg and I.H. Witten. Adaptive personalized interfaces: A question of viability. Behaviour and Information Technology, 4(1):31-45, 1985.

8. Richard Hazlett. Measurement of user frustration: a biologic approach. In CHI 'O3: CHI '03 extended abstracts on Human factors in computing systems, pages 734-735, New York, NY, USA, 2003. ACM. 
9. Anthony Jameson and Frank Wittig. Leveraging data about users in general in the learning of individual user models. In Proceedings of the 17th IJCAI, 2001.

10. Alfred Kobsa. User modeling: Recent work, prospects and hazards. In Matthias SchneiderHufschmidt, Uwe Malinowski, and Thomas Kuhme, editors, Adaptive User Interfaces: Principles and Practice, pages 111-128. Elsevier Science Inc., 1993.

11. Talia Lavie and Joachim Meyer. Benefits and costs of adaptive user interfaces. Int. J. Hum.-Comput. Stud., doi:10.1016/j.ijhcs.2010.01.004, 2010.

12. Regan L. Mandryk, Kori M. Inkpen, and Thomas W. Calvert. Using psychophysiological techniques to measure user experience with entertainment technologies. Behaviour $\&$ IT, 25(2):141-158, 2006.

13. Domen Novak, Matjaz Mihelj, and Marko Munih. Using psychophysiological measurements in physically demanding virtual environments. In INTERACT (1), pages 490-493, 2009.

14. Johanna Renny Octavia, Chris Raymaekers, and Karin Coninx. Investigating the possibility of adaptation and personalization in virtual environments. In G.J. Houben, G. McCalla, F. Pianesi, and M. Zancanaro, editors, UMAP 2009, volume 5535 of LNCS, pages 361-366. Springer, 2009.

15. Ivan Poupyrev, Mark Billinghurst, Suzanne Weghorst, and Tadao Ichikawa. The go-go interaction technique: non-linear mapping for direct manipulation in vr. In UIST '96: Proceedings of the 9th annual ACM symposium on User interface software and technology, pages 79-80, New York, NY, USA, 1996. ACM.

16. Ivan Poupyrev, Suzanne Weghorst, Mark Billinghurst, and Tadao Ichikawa. Egocentric object manipulation in virtual environments: Evaluation of interaction techniques. Comput. Graph. Forum, 17(3):41-52, 1998.

17. C. Rocchi, I. Graziola, D. Goren-Bar, O. Stock, and M. Zancanaro. Adaptive multimedia guide. In PEACH: Intelligent Interfaces for Museum Visits, pages 3-22. Springer-Verlag New York, Inc., 2007.

18. Lode Vanacken, Erwin Cuppens, Tim Clerckx, and Karin Coninx. Extending a dialog model with contextual knowledge. In TAMODIA 200\%, volume 4849 of $L N C S$, pages 28-41. Springer, 2007.

19. Lode Vanacken, Joan De Boeck, Chris Raymaekers, and Karin Coninx. Designing contextaware multimodal virtual environments. In ICMI 2008, pages 129-136, Chania, Crete, Greece, 2008.

20. Lode Vanacken, Tovi Grossman, and Karin Coninx. Multimodal selection techniques for dense and occluded 3d virtual environments. Int. J. Hum.-Comput. Stud., 67(3):237-255, 2009.

21. Chadwick A. Wingrave, Doug A. Bowman, and Naren Ramakrishnan. Towards preferences in virtual environment interfaces. In Proceedings of the 8th EGVE, pages 63-72, 2002. 